\title{
Metal-insulator (fermion-boson)-crossover origin of pseudogap phase of cuprates I: anomalous heat conductivity, insulator resistivity boundary, nonlinear entropy
}

\author{
B. Abdullaev ${ }^{1}$, C. -H. Park ${ }^{2}$, K. -S. Park ${ }^{3}$, I. -J. Kang ${ }^{4}$ \\ ${ }^{1}$ Institute of Applied Physics, National University of Uzbekistan, Tashkent 100174, Uzbekistan \\ ${ }^{2}$ Research Center for Dielectric and Advanced Matter Physics, Department of Physics, \\ Pusan National University, 30 Jangjeon-dong, Geumjeong-gu, Busan 609-735, Korea \\ ${ }^{3}$ Department of Physics, Sungkyunkwan University, 2066, Seoburo, Jangangu, Suwon, Gyeonggido, Korea \\ ${ }^{4}$ Samsung Mobile Display, Suwon, Kyunggido, Korea \\ bakhodir.abdullaeff@yandex.ru, cpark@pusan.ac.kr, pmpark@skku.edu, hacret@hanmail.net
}

PACS 74.72.-h, 74.20.Mn, 74.25.Fy, 74.25.Bt

DOI 10.17586/2220-8054-2017-8-1-48-58

\begin{abstract}
Among all the experimental observations of cuprate physics, the metal-insulator-crossover (MIC), seen in the pseudogap (PG) region of the temperature-doping phase diagram of copper-oxides under a strong magnetic field when the superconductivity is suppressed, is most likely the most intriguing one. Since it was expected that the PG-normal state for these materials, as for conventional superconductors, is conducting. This MIC, revealed in such phenomena as heat conductivity downturn, anomalous Lorentz ratio, insulator resistivity boundary, nonlinear entropy, resistivity temperature upturn, insulating ground state, nematicity- and stripe-phases and Fermi pockets, unambiguously indicates on the insulating normal state, from which high-temperature superconductivity (HTS) appears. In the present work (article I), we discuss the MIC phenomena mentioned in the title of article. The second work (article II) will be devoted to discussion of other listed above MIC phenomena and also to interpretation of the recent observations in the hidden magnetic order and scanning tunneling microscopy (STM) experiments spin and charge fluctuations as the intra PG and HTS pair ones. We find that all these MIC (called in the literature as non-Fermi liquid) phenomena can be obtained within the Coulomb single boson and single fermion two liquid model, which we recently developed, and the MIC is a crossover of single fermions into those of single bosons. We show that this MIC originates from bosons of Coulomb two liquid model and fermions, whose origin is these bosons. At an increase of doping up to critical value or temperature up to PG boundary temperature, the boson system undegoes bosonic insulator - bosonic metal - fermionic metal transitions.
\end{abstract}

Keywords: high critical temperature superconductivity, cuprate, metal-insulator-crossover, temperature-doping phase diagram, anomalous heat conductivity, insulator resistivity boundary, nonlinear entropy.

Received: 2 August 2016

Revised: 3 September 2016

\section{Introduction}

The origin of PG and HTS phases in copper oxides (cuprates) is one of the most puzzling and challenging problem in condensed matter physics. Despite being almost three decades since their discovery, intensive experimental and theoretical studies have yielded little clear understanding of these phases so far. The experimental studies of HTS and PG in cuprates have provided physicists with numerous interesting and fascinating materials with unconventional properties. Among the most puzzling and thus far most intriguing is the observation of the MIC, seen in the underdome region of a temperature-doping phase diagram in the absence [1-4] or presence [5-9] of a strong external magnetic field. The MIC, detected after suppression of the HTS by a strong magnetic field, results in a number of different phenomena: heat conductivity downturn and anomalous Lorentz ratio [10,11], insulator resistivity boundary [6], nonlinear entropy [12,13], insulating ground state (see Refs [1-4] and [5-9]), dynamic nematicity [14] and static stripe phases [15,16] and Fermi pockets [15-17]. This reveals the highly unconventional dielectric properties of the PG-normal phase of these superconductors. Since superconductivity appears 
in conventional superconductors from the conducting normal state only, the understanding of how HTS arises from an insulating state becomes a fundamental problem, and thus, the keystone for cuprate physics. This MIC also separates previously applied mechanisms and models for conventional superconductors from consideration.

Being previously introduced for the description of an electron transport transition of metals into insulators, as we see below in this and also in the second II papers, a concept of the MIC provides an opportunity to encompass various properties of the famous cuprate PG physics in the understanding within a single treatment. This treatment might be our Coulomb single boson and single fermion two liquid model, which was developed in Ref. [18].

Analyzing the electric charge and percolation of the visualized in STM real space PG and HTS nanoregions (NRs) of the $\mathrm{Bi}_{2} \mathrm{Sr}_{2} \mathrm{CaCu}_{2} \mathrm{O}_{8+\delta}$ compound [19,20] which exhibited an energy gap, we showed in [18] that NRs with minimal size are PG and HTS pairs and furthermore, they are single bosons, and percolation of these NRs occurs from the first critical doping, from which the HTS begins. In [18] we were also able to qualitatively understand all elements for the temperature-doping phase diagram of the copper-oxide. If the experiment [19] clearly showed, through the visualization of pair formation in the PG region, the precursor mechanism of the bulk HTS, we have successfully realized that the later appears due to percolative overlapping of the separated NRs. All positions of the Coulomb single boson and single fermion two liquid model are listed in Ref. [21]. This model is semi-phenomenological, since if the microscopic formation of single bosons, through the anyon bosonization of 2D fermions, is rigorously proven and after that experimentally confirmed in [18], then the size of these bosons and the percolative nature of the HTS are the results of Refs. $[19,20]$ data analysis.

In the present paper, we describe the low-temperature (low- $T$ ) non-Fermi liquid behavior of the heat-transport and entropy. As will be seen below, the central role in this calculation plays the temperature $T$ dependence of the electronic specific heat $c$. This $T$ dependence of $c$ is a result of the insulating ground state of 2D gas of plasmons, which consisted of charged single bosons, deformed and pinned by cuprate parent compound lattice ferroelectric atoms (the nematicity electronic state (see for details Ref. [21] and paper II)). Since, according to the Bogoliubov approach for gas of charged bosons [22], at high gas density, where this approach is valid, the ground state energy consists of components for the Bose-Einstein condensate and a gas of quasi-particle-plasmons. Following March et al. [23], who showed that the Bogoliubov approach can be nicely applied to the description of the liquid He II, we believe that this approach may at least qualitatively describe the intermediate densities of 2D Coulomb boson gas, at which the HTS takes place. At high magnetic fields or at lower dopings, close to the first critical doping, for which MIC phenomena are measured, the Bose-Einstein condensate vanishes and there only a gas of quasi-particle-plasmons exists. However, the latter, the individual charges of which being fixed by ferroelectric atoms, is insulating therefore, the insulator is the whole ground state of copper oxides. Typically, the experiment detects the MIC up to the critical value of doping [6-8], which, for some cuprates, is close to the optimal doping, where the $T_{c}$ is maximum in the temperature-doping phase diagrams. There also exists the case of $\mathrm{Bi}_{2} \mathrm{Sr}_{2} \mathrm{CaCu}_{2} \mathrm{O}_{8+\delta}$ compound [19], for which this critical doping almost coincides with the second critical doping, at which the HTS vanishes.

We start with the description of existing experimental data for the heat-transport and PG-normal state specific heat of copper-oxides. Hill and collaborators [10] reported that the heat conductivity of the electron doped copperoxide $\mathrm{Pr}_{2-x} \mathrm{Ce}_{x} \mathrm{CuO}_{4}$ measured at low- $T$ deviated from the one predicted by the Landau Fermi liquid theory (LFLT), i.e., as the temperature decreases, the temperature dependence of the heat conductivity $(\kappa)$ is changed from the normal linear $\kappa \sim T$ behavior into an anomalous $T^{3.6}$ one, which was described by the 'downturn' behavior of the heat conductivity. They also reported another important non-Fermi liquid behavior: the Lorentz ratio of the Wiedemann - Franz law (WFL) in the region of the linear $T$-dependence of $\kappa$ was significantly larger (1.7 times) than Sommerfeld's value. These violations were also observed in the heavily over-doped non-superconducting compound $\mathrm{La}_{2-x} \mathrm{Sr}_{x} \mathrm{CuO}_{4}$ by Nakamae et al. [24] and in $\mathrm{Bi}_{2+x} \mathrm{Sr}_{2-x} \mathrm{CuO}_{6+\delta}$ copper-oxide in the vicinity of the MIC by Proust et al. [11]. Smith et al. suggested that the downturn behavior of $\kappa$ results solely from the 
decoupling of the heat carrying thermal phonons and electrically conducting charge carriers [25] at low- $T$, while Hill et al. have indicated that the downturn of $\kappa$ should be intrinsic for copper-oxides (see Ref. [10]).

The normal state electronic specific heat $c$ of superconductors $\mathrm{YBa}_{2} \mathrm{Cu}_{3} \mathrm{O}_{6+x}$ and $\mathrm{La}_{2-x} \mathrm{Sr}_{x} \mathrm{CuO}_{4}$ above the HTS transition temperature $T_{c}$ was experimentally investigated in [12] and [13], respectively. A magnetic field dependence of $c$ has considered in [26] for $\mathrm{Y}_{0.8} \mathrm{Ca}_{0.2} \mathrm{Ba}_{2} \mathrm{Cu}_{3} \mathrm{O}_{6+x}$ compound. Due to existence of HTS, it is impossible to extract the information on the low- $T$ dependence of the normal state $c$. On the other hand, Loram et al. [12] showed the $T$-dependence of the entropy $(\mathcal{S}) \mathcal{S} \sim T^{i}$ with $i>1$ for the underdoped (insulating) material, which was driven from the measured electronic specific heat of HTS superconductors $\mathrm{YBa}_{2} \mathrm{Cu}_{3} \mathrm{O}_{6+x}$, ignoring the superconducting effects, while for the optimal doping compound, $\mathcal{S} \sim T$ was measured. The simple Drude model for the heat conductivity $\kappa$ will connect in our consideration the kinetic heat-transport property of PG region of HTS superconductors with its thermodynamic quantity - the electronic specific heat $c$. The entropy $\mathcal{S}$ is a result of $c$.

The low- $T$ MIC was observed firstly by Ando et al. [5] in the hole doped $\mathrm{La}_{2-x} \mathrm{Sr}_{x} \mathrm{CuO}_{4}$ compound through the measurement of the resistivity in $\mathrm{CuO}_{2}$ plane. This paper suggested the low- $T$ MIC is an intrinsic property rather than one induced by the $2 \mathrm{D}$ localization of carriers. The doping versus $T$ diagram of the insulating state up to near-optimal-doping was obtained in the extensive investigation by Boebinger et al. [6]. A similar (nearoptimal-doping) observation of the low- $T$ MIC was reported for the electron doped $\mathrm{Pr}_{2-x} \mathrm{Ce}_{x} \mathrm{CuO}_{4}$ by Fournier et al. [7] and for the 1/8-doped $\mathrm{Bi}_{2} \mathrm{Sr}_{2-x} \mathrm{La}_{x} \mathrm{CuO}_{6+\delta}$ by Ono et al. [8]. The low-T MIC were clearly measured in a La-free hole-doped $\mathrm{Bi}_{2+x} \mathrm{Sr}_{2-x} \mathrm{Cu}_{1+y} \mathrm{O}_{6+\delta}$ of the doping $x=0.13$ (while optimal doping was $x=0.16$ ) by Vedeneev and Maude [27] through the measurement of the in-plane resistivity. Although in some papers (see, for example, Ref. [27]) authors tried to invoke the 2D carrier localization approach to explain the phenomenon, the material-independent and universal character of the low- $T$ MIC for all cuprates indicates that effect is intrinsic and may be related to a fundamental property of cuprates.

It was already mentioned above that according to Ref. [25] the downturn of the heat conductivity is related to a decoupling of charges and phonons. However, the paper [25] does not clarify the origin of this decoupling. Observations of the low- $T$ MIC, and the downturn for the same compound and under same experimental conditions are not occasional and indicate on the MIC origin of the decoupling, when charges localized in the insulating state cease to do a scattering with phonons. Therefore, the non-linear insulating $\mathcal{S} \sim T^{i}$ with $i>1$ dependence of entropy, the downturn behavior $\kappa \sim T^{3.6}$ and the low- $T$ MIC are results of the same underlying physics of the insulating ground state and MIC for cuprates.

In this work, we demonstrate that the unified description of the three MIC phenomena is possible. In Sec. 2, we derive the specific heat of the ideal gas of Bogoliubov quasi-particles (IGBQ) and demonstrate that it is obtained from the contributions of plasmons at low- $T$ and free quasi-particles at high- $T$ temperatures. We will find in the section $T$, at which one part of the specific heat transits into another one. In Sec. 3, the low- $T$ dependence and the downturn temperature of the heat conductivity $\kappa$ will be determined. In this section, we also obtain the Lorentz ratio of the WFL and discuss the arguments supporting our approach for the explanation of the observed insulating ground state (IGS) and low-T MIC. Analysis of the experimental data on the specific heat for copper-oxides and their possible relation to $c$ described in the present paper will be made in Sec. 4. In this section we obtain also the expression for entropy $\mathcal{S}$. We conclude and summarize the paper by Sec. 5 .

\section{Specific heat of IGBQ}

As was indicated in the first section, the ground state for the low dopings of cuprates is HTS from charged bosons. However, under strong magnetic fields, which were used for the measurements of the heat conductivity $\kappa$ and the IGS and low- $T$ MIC, the HTS is suppressed and for PG normal state at low- $T$ we have only the excited 
state - IGBQ, which is not effected by magnetic field (see Sec. 4 below). Therefore, in order to investigate the $T$ dependence of $c$ for this low doping and low- $T$, we calculate the specific heat of IGBQ.

The thermodynamic free energy [28] of an ideal boson gas is:

$$
F=T \int \frac{d^{2} p d^{2} x}{(2 \pi \hbar)^{2}} \ln \left(1-e^{-\varepsilon(p) / T}\right)
$$

Here, the temperature $T$ is described by the energy scale. We use a relation between the quasi-particle (QP) energy $\varepsilon$ and momentum $p$ of IGBQ, which was derived previously [22]: $\varepsilon(p)=\left(a p+\left(p^{2} / 2 m\right)^{2}\right)^{1 / 2}$, where ap is the square of QP energy of plasmon, and $a=2 \pi e^{2} n \hbar / m$. Here, $e$ and $n$ are the charge and 2D density, respectively, of real particles with mass $m$.

One can separate the integral of Eq. (1) over the absolute value of momentum $p$ into two parts: $\int_{0}^{\infty}=$ $\int_{0}^{q}+\int_{q}^{\infty}$. Here $q$ gives the maximum of the momentum distribution function of non-condensate real particles [29] at $T=0$ under the Bogoliubov approximation. Thus, the contributions of the plasmon (dominant for $p<q$ ) and of the kinetic energy (dominant for $p>q$ ) to the energy of QPs are approximately separated at the boundary $(p=q)$, where:

$$
(a q)^{1 / 2}=q^{2} /(2 m)
$$

For the first (second) integral, if we introduce the variable $x=(a p)^{1 / 2} / T\left(x=p^{2} /(2 m T)\right)$, we obtain:

$$
F \approx \frac{T S}{2 \pi \hbar^{2}}\left[\frac{2 T^{4}}{a^{2}} \int_{0}^{(a q)^{1 / 2} / T} d x x^{3} \ln \left(1-e^{-x}\right)+m T \int_{(a q)^{1 / 2} / T}^{\infty} d x \ln \left(1-e^{-x}\right)\right]
$$

where $S$ is defined to be the area of $2 D$ system. (i) At lower temperatures, one can have $(a q)^{1 / 2} / T \gg 1$, and one can replace the upper limit of first integral in Eq. (3) by infinity sign, and the second integral becomes zero. (ii) At high temperature, we can have $(a q)^{1 / 2} / T \ll 1$, then the lower limit of second integral can be replaced by zero and the first integral is removed. Following the calculational scheme of Ref. [28], we thus find that the specific heat per area can be expressed at low- $T$ by:

$$
c_{1}=-\frac{20 T^{4} A}{\pi \hbar^{2} a^{2}}
$$

with the ideal gas of the plasmons and at high- $T$ by:

$$
c_{2}=-\frac{T m B}{\pi \hbar^{2}}
$$

with the ideal gas of free QPs. Here,

$$
A=-(1 / 4) \int_{0}^{\infty} x^{4} d x /\left(e^{x}-1\right)=-(1 / 4) \Gamma(5) \zeta(5) \quad \text { and } \quad B=-\int_{0}^{\infty} x d x /\left(e^{x}-1\right)=-\Gamma(2) \zeta(2),
$$

where $\Gamma(y)$ and $\zeta(y)$ are Gamma and the Riemann Zeta functions, respectively.

We define a temperature $T_{d}^{c}$, at which the low- $T$ dependence of $c_{1}$ transforms into that of $c_{2}$ at high- $T$. It can approximately be determined by setting equal the approximate equations for $c_{1}$ and $c_{2}$. It has the expression:

$$
T_{d}^{c}=\left(\frac{\Gamma(2) \zeta(2)}{5 \Gamma(5) \zeta(5)}\right)^{1 / 3}\left(m a^{2}\right)^{1 / 3}
$$

One can show that $\left(m a^{2}\right)^{1 / 3}=2\left(2 / r_{s}^{2}\right)^{2 / 3} R y$, where $r_{s}$ is the mean distance between particles in Bohr $a_{B}$ radius $\left(a_{B}=\hbar^{2} /\left(m e^{2}\right)\right)$ and $R y$ is Rydberg $\left(R y=m e^{4} /\left(2 \hbar^{2}\right)\right)$ energy. We use the relation [30,31] $n_{a b}=$ $6.747 \cdot 10^{14} \mathrm{t} / \mathrm{cm}^{2}$ between the density of charges in cuprate $1 \mathrm{~cm}^{2}$ area of $a-b$ plane and fraction $t$ of charge per $\mathrm{Cu}$ atom. Expressing the density in $1 / a_{B}^{2}$ units we find $r_{s}$ as function of $t$. Therefore, $T_{d}^{c}$ is:

$$
T_{d}^{c} \approx 0.114 t^{2 / 3} R y \text {. }
$$


The specific heat at an arbitrary temperature is obtained from Eq. (1), which has the form:

$$
c=\frac{q^{2}}{2 m} \frac{m}{4 \pi \hbar^{2}} \int_{0}^{\infty} \frac{d y}{z^{2}} \frac{y^{2}\left(1+y^{3}\right)}{\sinh ^{2}\left(y^{1 / 2}\left(1+y^{3}\right)^{1 / 2} /(2 z)\right)},
$$

where $z=T /(a q)^{1 / 2}$ and $y=p / q$. Fig. 1 shows the $c / z$ (in $q^{2} /\left(8 \pi \hbar^{2}\right)$ units) calculated by using Eq. (8). Here, it is remarkable that the curve can nicely describe the downturn behavior of the specific heat. The downturn occurs around $z=0.5$, indicating that $T_{d}^{c}=0.5(a q)^{1 / 2}$. Here, $(a q)^{1 / 2}=8\left(2^{1 / 2} /\left(3 r_{s}^{2}\right)\right)^{2 / 3} R y$ and expressing $r_{s}$ through $t,(a q)^{1 / 2}=0.737 \cdot t^{2 / 3} R y$. By the numerical estimation of the downturn temperature $T_{d}^{c}$, the coefficient in Eq. (7) becomes 0.368 instead of 0.114 .

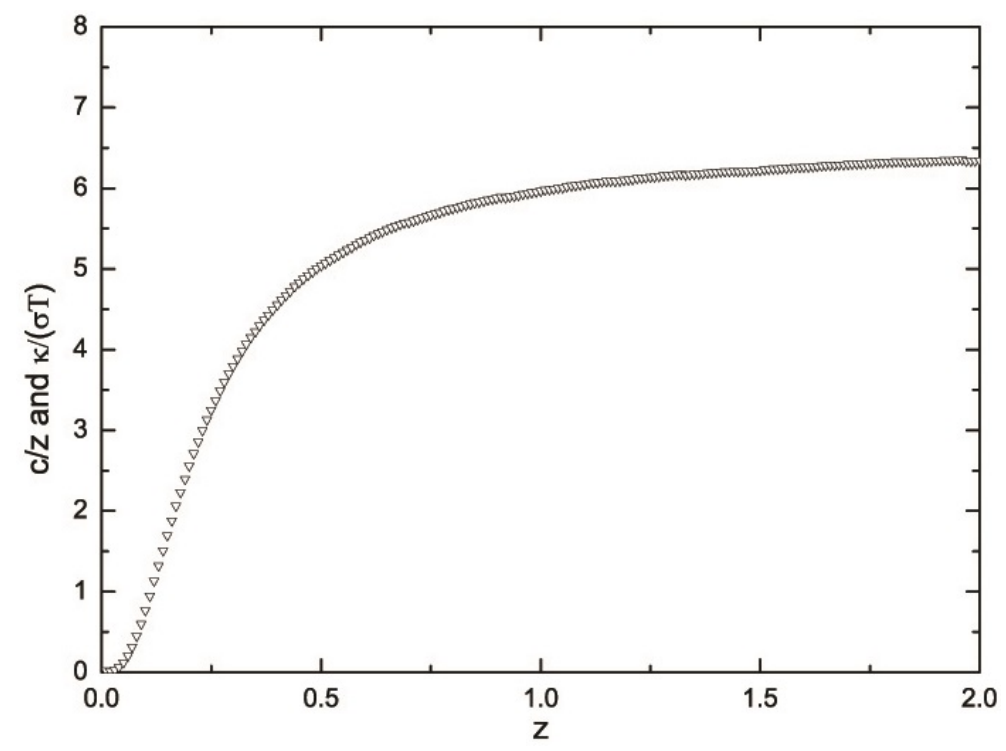

FIG. 1. The specific heat $c / z$ (in $q^{2} /\left(8 \pi \hbar^{2}\right)$ units) and $\kappa /(\sigma T)$ (in $k_{B} m v l /\left(2 \pi \hbar^{2} \sigma\right)$ units) as function of $z$. The definition of $z: z=T /(a q)^{1 / 2}$ for $c / z$ and $z=K^{1 / 3} T /(a q)^{1 / 2}$ for $\kappa /(\sigma T)$, where $K \sim 10^{12}$ (see text)

\section{Heat conductivity and MIC}

The downturn behavior for the heat conductivity $\kappa$ deviated from the WFL can be explained by a crossover of freely penetrating QPs of IGBQ into localizing individual particles collective plasmon state. As mentioned in the first section, the $a-b$ plane cuprate atoms deform the intra charge structure of single bosons and pin them (fixed nematicity phase). However, they become free (free QPs) at the bosonic insulator - bosonic metal transition temperature and doping (see below). At the same time, despite the fact that the charged single bosons are pinned before this transition, the plasmon gas is free for a penetrating.

The plasmon gas, which becomes dominant at low- $T$, does not carry the charge and is electrically insulating, therefore the formation of plasmons at low- $T$ significantly affects the behavior of $\kappa$. One can approximately estimate the downturn temperature $T_{d}^{\kappa}$ of $\kappa$ as a temperature, where WFL of electrically conducting free QPs with electrical conductivity $\sigma_{2}$ transits into WFL of plasmons (with the electrical conductivity $\sigma_{1}$ ). We apply the WFL in the region of this nearly insulating gas (mainly consisted of plasmons) to calculate $\sigma_{1}$ from the $\kappa$ and specific heat. Although the WFL is not conventionally applicable for insulators, we will demonstrate in this section that in the case of cuprates $\sigma_{1}$ for plasmons equals $\sigma_{2}$ of conductor with infinitely small numerical prefactor. Thus for plasmon gas as conductor with very small electrical conductivity the WFL is formally valid. On the other hand, this "bad" conductor satisfies the formal definition of insulator given in the classical electrodynamics as a 
conductor having its conductivity close to zero. While the Lorentz ratio in our paper calculates only for free QPs of IGBQ, i.e., for conductor above the downturn $T_{d}^{\kappa}$ temperature. We cannot, strictly saying, apply the WFL for insulating plasmon gas to calculate the Lorentz ratio. This our result for Lorentz ratio, Fig. 2 is in correspondence with experiment of Proust et al. [11].

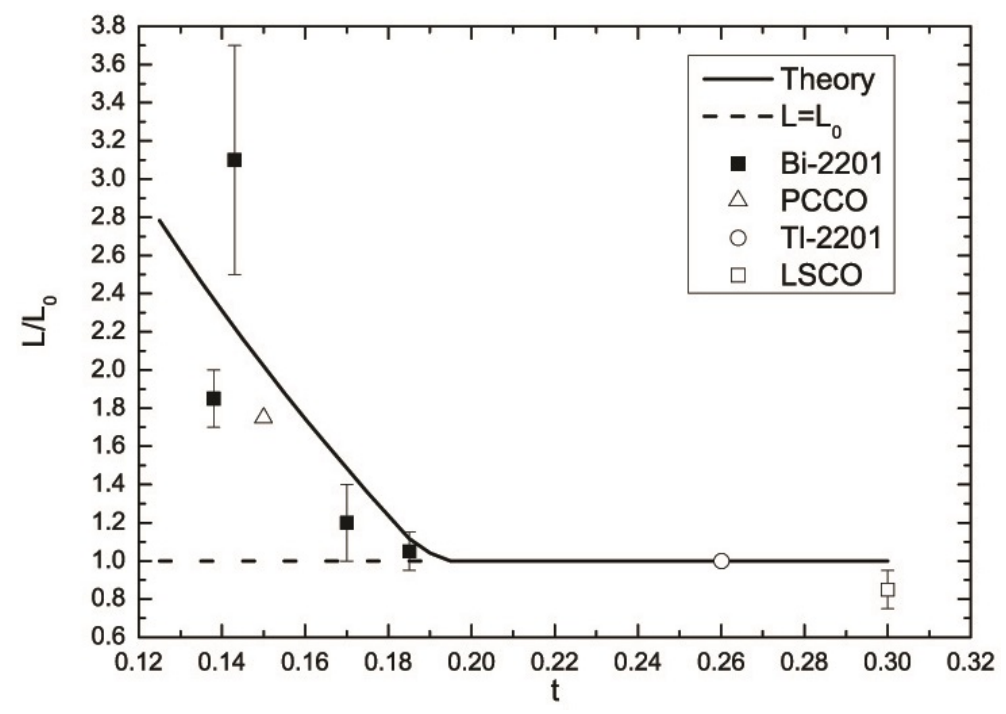

FIG. 2. The Lorentz ratio $L / L_{0}$ (Eq. (14)) vs. $t$ (values for $t \geq 1$ are added artificially).

Observed dots are from Ref. [11]

According to the Drude model, which we will apply for the description of heat conductivity $\kappa$, only two quantities (except the specific heat $c$ ) contribute in $\kappa$ (see below): mean velocities and free penetration lengths of QPs. For the low- $T$ regime, at which the experiments on $\kappa$ are measured, one can ignore the $T$ dependence of these two (if the mean velocity is determined by concentration of charges, then the free penetration length is determined for low- $T$ by scattering of charges on impurities). Therefore, the qualitative $T$ dependence of $\kappa$ (together with downturn $T_{d}^{\kappa}$ ) is determined only by the specific heat $c$.

Following the Drude model, we have $\kappa_{1}(T)=(1 / 2) c_{1}(T) v_{1} l_{1}$ for gas of plasmons and $\kappa_{2}(T)=(1 / 2) c_{2}(T) v_{2} l_{2}$ for gas of free QPs with corresponding mean velocities and free penetration lengths $v_{1,2}$, and $l_{1,2}$, respectively. To obtain the value of $T_{d}^{\kappa}$, we express the specific heat Eq. (8):

for $z \ll 1$ and

$$
c_{1}=\frac{q^{2}}{2 m} \frac{m}{\pi \hbar^{2}} z^{4} 10 \Gamma(5) \zeta(5)
$$

$$
c_{2}=\frac{q^{2}}{2 m} \frac{m}{\pi \hbar^{2}} z \Gamma(2) \zeta(2)
$$

for $z \gg 1$. Substituting $z=T /(a q)^{1 / 2}$ and using Eq. (2), it is found that Eq. (9) reduces to Eq. (4) and Eq. (10) to Eq. (5).

Therefore, WFL is expressed as:

$$
\frac{\kappa_{1}}{\sigma_{1} T}=\frac{k_{B} m v_{1} l_{1}}{2 \pi \hbar^{2} \sigma_{1}} z^{3} 10 \Gamma(5) \zeta(5)
$$

for gas of plasmons and

for gas of free QPs.

$$
\frac{\kappa_{2}}{\sigma_{2} T}=\frac{k_{B} m v_{2} l_{2}}{2 \pi \hbar^{2} \sigma_{2}} \Gamma(2) \zeta(2)
$$

At the downturn temperature $T_{d}^{\kappa}$, it should be $\kappa_{1}\left(T_{d}^{\kappa}\right) /\left(\sigma_{1} T_{d}^{\kappa}\right)=\kappa_{2}\left(T_{d}^{\kappa}\right) /\left(\sigma_{2} T_{d}^{\kappa}\right)$. However, due to the nonequality $v_{1} l_{1} / \sigma_{1} \neq v_{2} l_{2} / \sigma_{2}$, we cannot express Eq. (11) and Eq. (12) as single function of WFL, which transforms 
from low- $T$ limit into high- $T$ limit with the increase of $T$. On the other hand, if we write $v_{1} l_{1} / \sigma_{1}=K_{\sigma_{1}, \sigma_{2}} v_{2} l_{2} / \sigma_{2}$, where $K_{\sigma_{1}, \sigma_{2}}$ is numerical factor, and if we introduce the definition $v l / \sigma=v_{2} l_{2} / \sigma_{2}$ for gas of free QPs, the single WFL for new parameter can be expressed by $z=K_{\sigma_{1}, \sigma_{2}}^{1 / 3} T /(a q)^{1 / 2}$. This can describe the WFL of the free QPs gas at all $T$. In Fig. 1, we plot the $\kappa /(\sigma T)$, (expressed in $k_{B} m v l /\left(2 \pi \hbar^{2} \sigma\right)$ units) as function of $z$, which is valid at low- $T$, where $v, l$ and $\sigma$ are $T$ independent.

We estimate the electrical conductivity $\sigma_{1}$ for a case of $t \sim 0.1$. From the expression for $(a q)^{1 / 2}$, one derives $(a q)^{1 / 2} / k_{B} \sim 10^{4} \mathrm{~K}$ (in $\mathrm{K}-$ Kelvin temperature units). Thus, the downturn of the specific heat takes place at $T_{d}^{c} \sim 10^{4} \mathrm{~K}$. The observed downturn- $T$ of the heat conductivity $[10,11]$ is $T_{d, \text { exp }}^{\kappa} / k_{B} \sim 0.1 \mathrm{~K}$. By using the downturn coordinate $z=0.5$ from Fig. 1 for $\kappa /(\sigma T)$ and by substituting in this $z$ values of $(a q)^{1 / 2} / k_{B}$ and $T_{d, \text { exp }}^{\kappa} / k_{B}$, we obtain $K_{\sigma_{1}, \sigma_{2}} \sim 10^{12}$. This means that $\sigma_{1} v_{2} l_{2} /\left(\sigma_{2} v_{1} l_{1}\right) \sim 10^{-12}$. We compare $v_{1}$ with $v_{2}$ and $l_{1}$ with $l_{2}$. Free QPs crossover into plasmons at momentum $q$ determined by Eq. (2). However, this expression for $q$ is similar to the expression for the critical momentum for the Landau damping of electrons [32], which decay plasmons being generated or absorbed. Thus, we can apply the Landau damping approach in our case. However, except an energy conservation law - Eq. (2), this approach requires an equality of the plasmon phase velocity and electron velocity. Therefore, for an estimate, one can assume at $T_{d}^{\kappa}$ that $v_{1} \sim v_{2}$ and $l_{1} \sim l_{2}$ (here we take that the plasmon phase velocity is roughly equal to the plasmon group velocity and the lifetime of plasmon and electron against to decay is the same), i.e., mean velocities and free penetration lengths for plasmons and free QPs have the same order of magnitude. Hence, we obtain $\sigma_{1} / \sigma_{2} \sim 10^{-12}$. This result supports the above supposed assumption of the IGS, in which the insulating plasmon gas formed from the charge conducting free QP gas transits at low- $T$.

This analysis allow us to suggest that the downturn of heat conductivity may be a result of the MIC at low- $T$. In addition to coincidence of experimental parameters for the doping and of magnetic field strength at the measurements of $\kappa$ and the IGS, and low-T MIC for the same copper-oxide, as discussed above, the MIC boundary described by Boebinger et al. (see Ref. [6]) (at higher $T$ ) has qualitatively the same doping dependence as the PG boundary. In Ref. [30], we limited the existence region of anyon-related Coulomb interacting bosons below the PG boundary because close to PG boundary bosons were transformed to fermions. This observation of Boebinger et al. supports the frame of our approach on the nature of $\mathrm{PG}$ phase as a mixture of single particle bosons and normal fermions. In our description the region below Boebinger et al. MIC boundary is dominated by plasmons, while close and right above of this boundary by free QPs of IGBQ.

This scenario for MIC corresponds to the bosons of the Coulomb single boson and single fermion two liquid model and fermions, whose origin is these bosons. At an increase of doping up to a critical value or temperature up to PG boundary temperature, this boson system undergoes bosonic insulator - bosonic metal - fermionic metal transitions. While there also exists a small part of the model fermion component, which is not related to single bosons and at the same variation of doping or temperature undergoes insulator - metal crossover $[18,21]$ and does not contribute to the MIC.

We determine the Boebinger et al. MIC boundary for temperature and doping in the doping-temperature phase diagram. This boundary defines temperature as function of doping, at which bosonic insulator - bosonic metal transition occurs. The analytic form for the transition temperature is expressed by Eq. (7) with coefficient 0.368 instead of 0.114 for the downturn temperature $T_{d}^{c}$ of the specific heat. In the next paper, part II, we will demonstrate that the low- $T$ dependence of a resistivity is determined by the specific heat. However, in Eq. (7), we used the expression for the entire density of charges $n_{a b}$. While one needs to separate in it the contributions from single boson and single fermion parts. We write the formula $n_{a b}=n_{a b}\left(1-t / t_{c}\right)+n_{a b} t / t_{c}$, in which the first term describes the approximate single boson density and the second one the approximate single fermion density. Our interest is in the single bosons therefore, the expression for the bosonic insulator - bosonic metal transition 
temperature is:

$$
T_{b I M} \approx 0.368\left[t\left(1-\frac{t}{t_{c}}\right)\right]^{2 / 3} R y .
$$

Eq. (13) describes the temperature and doping boundary for the MIC of resistivity, found in the experiment of Boebinger et al. [6].

We now calculate the Lorentz ratio for free QPs of IGBQ. The WFL, $\kappa /(\sigma T)=L_{0}$, of heat transport for QPs of LFLT can be expressed by the Lorentz ratio $L_{0}$ and Sommerfeld's value $L_{0}=\left(\pi^{2} / 3\right)\left(k_{B} / e\right)^{2}$. We note that this value of $L_{0}$ corresponds to the three dimensional (3D) case. A simple calculation shows that it can be also applied for 2D case. For the IGBQ, it can be assumed that the mean kinetic energy of QPs is $m v^{2} / 2=(a q)^{1 / 2}$ (Eq. (2), because, as was pointed out above, at $T=0$ the most part of non-condensate particles has this energy). Then $\kappa /(\sigma T)=m v^{2} c /\left(2 n e^{2} T\right)$, with $c$ determined from Eq. (5) for free QPs of IGBQ, reduces to a form $\kappa /(\sigma T)=3.106 \cdot L_{0} / t^{1 / 3}$. This expression corresponds to the WFL of bosons, when there is no mixture of bosons with Fermi QPs of LFLT. However, at the increasing of concentrations of holes or electrons $t$ to the direction of the critical doping $t_{c}$, fermions appear in the PG region [30] and this mixture occurs. Therefore, at $T \rightarrow 0$ we can phenomenologically write the expression $\kappa /(\sigma T)=L$ with:

$$
L=L_{0}\left[\frac{3.106}{t^{1 / 3}}\left(1-\frac{t}{t_{c}}\right)+\frac{t}{t_{c}}\right],
$$

which takes into account the transformation of $L$ from one of free QPs of IGBQ to one of LFLT QPs, when the concentration $t$ tends to (but below) critical concentration $t_{c} \approx 0.19$ [33]. In Fig. 2, we compare our estimated $L / L_{0}$ with the experimental data taken from Fig. 7 (a) of [11] for several cuprate compounds. It is remarkable that the calculated curve is in good agreement with the observed values of $L / L_{0}$.

\section{Specific heat and entropy}

The qualitative features $[12,13,33]$ of the observed cuprate electronic specific heat and entropy are as follows. The increment coefficient $\gamma=c / T$ of the specific heat in the HTS-to-normal-state phase transition (PT) point has weakly apparent and washed out peak in the lightly underdoped side. The peak of $\gamma$ and its form become higher and sharper, respectively, when $t$ increases. Peak is maximal (with the sharpest form) at $t_{c}$ and then does not almost change the form for $t>t_{c}$. There is no influence on the normal state $c$ by the external magnetic field [26]. At last, $\gamma$ is independent from doping for $t>t_{c}$ [12].

In general, the weakly apparent peak of $\gamma$ is attributed [28] to the first order PT, which is close to the second order one. Sharpening of $\gamma$ at $t_{c}$ might characterize the transition of PT into the second order type, where conventional superconductivity scenario with Cooper pairs becomes effective. The hypothesis in accordance with this description was pointed out in [30].

The non-dependence of the normal state $c$ on the magnetic field might be a result that QP energy $\varepsilon(p)$ of IGBQ, by analogy with Cooper pair energy $\varepsilon_{C p}(p)$, is independent of magnetic field. Abrikosov [34] has shown that $\varepsilon_{C p}(p)$ being the result of canonical Bogoliubov transformation is function of scalar quantities $u_{p}$ and $v_{p}$. However, the latter ones, as scalars, in the magnetic field with gauge $\vec{\nabla} \cdot \vec{A}(\vec{r})=0$ can be function of only zero scalar product $\vec{k} \cdot \vec{A}_{\vec{k}}=0$, where $\vec{k}$ and $\vec{A}_{\vec{k}}$ are Fourier transforms of vector-coordinate $\vec{r}$ and vector-potential $\vec{A}(\vec{r})$, respectively. The independent from the magnetic field PG normal state was also observed in the resistivity measurement of the MIC [35], thus sustaining the possible role of $\varepsilon(p)$ of IGBQ.

For the specific heat $c_{F}$ of 2D gas of LFLT QPs one obtains the same linear $T$ dependence as in Eq. (5), but with ratio $c_{F} / c_{2}=2$ (due to two directions of spins for fermions instead of bosons). The non- $t$-dependence observation of $\gamma$ for $t>t_{c}$ might be a result of the non-concentration dependence for Eq. (5). 
We obtain the expression of the electronic normal state entropy $\mathcal{S}$ through the calculation of integral $\mathcal{S}=\int_{0}^{T}\left(c / T_{1}\right) d T_{1}$ for $\mathrm{PG}, T \leq T^{*}$, region. Here $T^{*}$ is temperature of PG boundary. Typical experimental scale of $T^{*}$ is $T^{*} / k_{B} \sim 10^{2} \mathrm{~K}$, while $(a q)^{1 / 2} / k_{B} \sim 10^{4} \mathrm{~K}$, therefore, in Eq. (8) one can assume $z \ll 1$ and use Eq. (4) as approximate expression of the specific heat of IGBQ. For arbitrary $T$ (in the interval of $T \leq T^{*}$ ), we write the phenomenological expression:

$$
\frac{c}{T}=\frac{c_{1}}{T}\left(1-\frac{5}{4} \frac{T}{T^{*}}\right)+\frac{2 c_{F}}{T} \frac{T}{T^{*}} .
$$

The factor $5 / 4$ in front of first $T / T^{*}$ term was introduced for convenience purpose. The integration over $T_{1}$ gives:

$$
\mathcal{S}=\frac{c_{1}}{4}\left(1-\frac{T}{T^{*}}\right)+c_{F} \frac{T}{T^{*}} .
$$

We compare the $T$ dependence of our $\mathcal{S}$ with experimental one of [12]. It is convenient to express $\mathcal{S}$ in $\mathrm{mJ} /\left(\mathrm{mol} \mathrm{K}\right.$ ) units and $T$ in $\mathrm{K}$ units (we use the approximate PG boundary $T^{*}=900-4736.8421 \cdot t$ taken from Fig. 11 of Ref. [33] and at calculating of $\mathcal{S}$ we assume that value of charge is $t$ ). In this case, the increment coefficient $\gamma$ from Fig. 4 of [12], being multiplied by $T$, gives $\mathcal{S}$ as function of $T$, analogous to Fig. 6 of [26]. However, if Fig. 6 describes only the metallic $t$ of holes, we obtain the data and for the insulating $t$. Comparing in Fig. 3 the plot of $\mathcal{S}$ obtained from Eq. (16) with one from Fig. 6 of [26] we see (i) the general nonlinear, $\sim T^{i}$ with $i>1$, behavior of all curves for $t<t_{c}$, (ii) all curves for $T>T^{*}$ have a linear behavior with the same slope, $\gamma \approx 1.46$, as for $2 \mathrm{D}$ fermion gas, (iii) in contrast to experimental result our curves for $T>T^{*}$ are not parallel. However, the parallelism of the observed curves indicated in the last point is inconsistent with the clear tendency of $\gamma$ to approach the fixed value as $T$ goes to infinity (more obviously it is seen in [13] for $\mathrm{La}_{2-x} \mathrm{Sr}_{x} \mathrm{CuO}_{4}$ compound, although, in this paper $\gamma \approx 1.0$ was obtained). Finally, the alternative $T / T^{*}$ dependencies are considered in [36] for $T$ just after $T_{c}$ of HTS and near the optimal doping, and in [37] close to $T^{*}$.

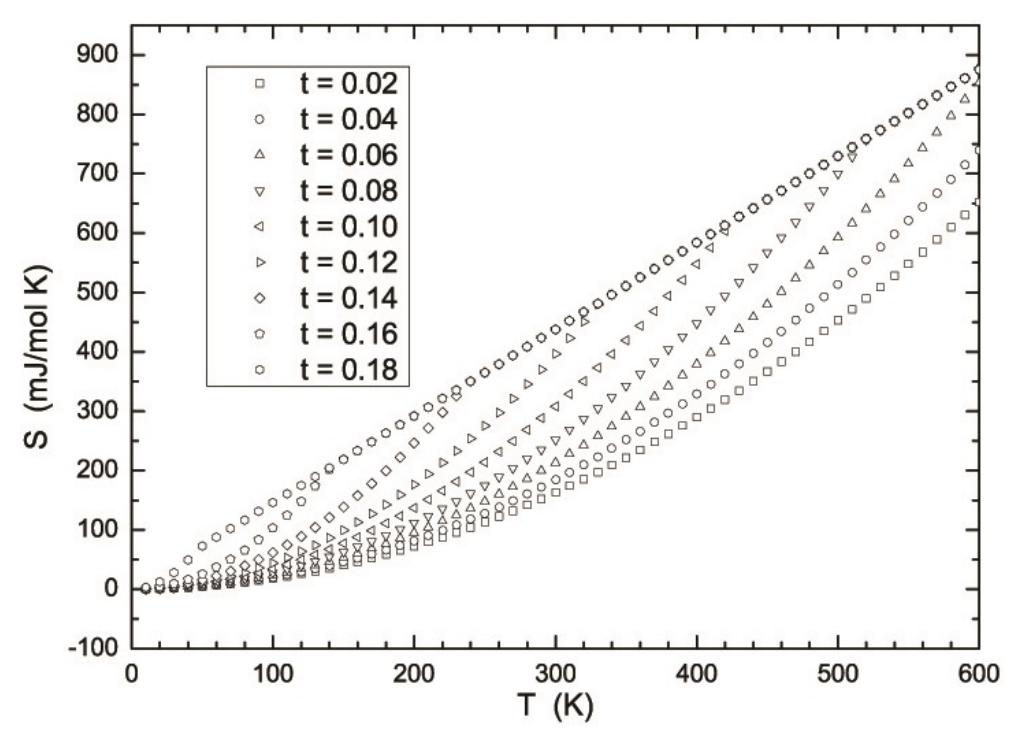

FIG. 3. The entropy $\mathcal{S}$ (Eq. (16)) vs. $T$ at various $t$ (values of $\mathcal{S}$ behind the crossing of linear and nonlinear parts of $\mathcal{S}$ are added artificially).

However, irrespective the quality of $t / t_{c}$ and $T / T^{*}$ laws in Eqs. (14) and (16) one can say that at small values for these parameters the single particle boson contribution into $\kappa$ and $\mathcal{S}$ properly describes the experiment. 


\section{Summary and conclusion}

We have carried out an attempt to describe in the unified anyon-related boson approach the PG phase electronic low- $T$ heat conductivity $\kappa$, entropy $\mathcal{S}$ and the IGS, and low- $T$ MIC of cuprates. We have argued that the observed $\kappa \sim T^{3.6}$ and $\kappa \sim T$ is a result of $c \sim T^{4}$ and $c \sim T$ dependencies, respectively, while $\mathcal{S} \sim T^{i}$ with $i>1$ originates from $c \sim T^{4}$ of the specific heat $c$ for IGBQ of 2D Coulomb-interacting boson gas. Providing by the qualitative and quantitative arguments, we have attributed the downturn behavior of $\kappa$ to the MIC and transition into the IGS. Assuming that the PG phase consisted from the mixture of IGBQ and 2D LFLT QP gas, we have obtained the Lorentz ratio values of WFL, which were close to experimental ones. We have clarified the origin of the decoupling of charge carriers with phonons as transition of free QPs of IGBQ into plasmons.

We have shown that the total MIC originates from bosons of the Coulomb single boson and single fermion two liquid model and fermions, whose origin is these bosons. At an increase of doping up to the critical value (Eq. (14)) or temperature up to PG boundary temperature (Eq. (16)), this boson system undergoes bosonic insulator - bosonic metal - fermionic metal transitions. It is interesting that this MIC behavior of the doping and temperature resembles the right-angled triangle rule, in which variables vary along horizontal and vertical legs, respectively. We note that there also exists a small part of the model fermion component, which is not related to single bosons and at the same variation of doping or temperature undergoes insulator - metal crossover and does not contribute to the MIC (see Ref. [18]). We have succeeded in describing of the Boebinger et al. experimental temperature and doping MIC boundary of resistivity.

The good agreement of the calculated within Coulomb two liquid model results for heat conductivity downturn, anomalous Lorentz ratio, and nonlinear entropy with experimental ones may indicate on the correctness of this model's concepts. In paper II of this series, we will try to understand within our model the physics of other MIC phenomena: resistivity temperature upturn, insulating ground state, nematicity- and stripe-phases, and Fermi pockets. The next important issue obtained in paper II will be a demonstration that the recently observed in the hidden magnetic order and STM experiments spin and charge fluctuations are the intra PG and HTS pair fluctuations.

Some results of the presented paper, without derivation and detail discussion, have partially been published in Ref. [21].

\section{Acknowledgement}

Authors B. Abdullaev and C.-H. Park acknowledge the support of the research by the National Research Foundation (NRF) Grant (NRF-2013R1A1A2065742) of the Basic Science Research Program of Korea.

\section{References}

[1] Takagi H. et al. Systematic evolution of temperature-dependent resistivity in $\mathrm{La}_{2-x} \mathrm{Sr}_{x} \mathrm{CuO}_{4}$. Phys. Rev. Lett., 1992, 69, P. $2975-2979$.

[2] Keimer B. et al. Magnetic excitations in pure, lightly doped, and weakly metallic $\mathrm{La}_{2} \mathrm{CuO}_{4}$. Phys. Rev. B, 1992, 46, P. 14034.

[3] Wuyts B., Moshchalkov V.V., Bruynseraede Y. Resistivity and Hall effect of metallic oxygen-deficient $\mathrm{YBa}_{2} \mathrm{Cu}_{3} \mathrm{O}_{x}$ films in the normal state. Phys. Rev. B, 1996, 53, P. 9418.

[4] Abe Y. et al.Normal-state magnetotransport in $L a_{1.905} \mathrm{Ba}_{0.095} \mathrm{CuO}_{4}$ single crystals. Phys. Rev. B, 1999, 59, P. 14753.

[5] Ando Y. et al. Logarithmic Divergence of both In-Plane and Out-of-Plane Normal-State Resistivities of Superconducting $\mathrm{La}_{2}-x \mathrm{Sr}_{x} \mathrm{CuO}_{4}$ in the Zero-Temperature Limit. Phys. Rev. Lett., 1995, 75, P. 4662-4665.

[6] Boebinger G. S. et al. Insulator-to-Metal Crossover in the Normal State of $\mathrm{La}_{2-x} \mathrm{Sr}_{x} \mathrm{CuO}_{4}$ Near Optimum Doping. Phys. Rev. Lett., 1996, 77, P. 5417-5420.

[7] Fournier P. et al. Insulator-Metal Crossover near Optimal Doping in $\operatorname{Pr}_{2-x} \mathrm{Ce}_{x} \mathrm{CuO}_{4}$ : Anomalous Normal-State Low Temperature Resistivity. Phys. Rev. Lett., 1998, 81, P. 4720-4723.

[8] Ono S. et al. Metal-to-Insulator Crossover in the Low-Temperature Normal State of $\mathrm{Bi}_{2} \mathrm{Sr}_{2-x} \mathrm{La}_{x} \mathrm{CuO}_{6+\delta}$. Phys. Rev. Lett., 2000, 85, P. 638-641. 
[9] Ando Y. et al. Supporting evidence of the unusual insulating behavior in the low-temperature normal-state resistivity of underdoped $\mathrm{La}_{2-x} \mathrm{Sr}_{x} \mathrm{CuO}_{4}$. J. Low Temp. Phys., 1996, 105, P. 867-875.

[10] Hill R. W. et al. Breakdown of Fermi-liquid theory in a copper-oxide superconductor. Nature, 2001, 414, P. 711-715.

[11] Proust C. et al. Heat transport in $\mathrm{Bi}_{2+x} \mathrm{Sr}_{2-x} \mathrm{CuO}_{6+\delta}$ : Departure from the Wiedemann-Franz law in the vicinity of the metal-insulator transition. Phys. Rev. B, 2005, 72, P. 214511.

[12] Loram J. W. et al. Electronic specific heat of $\mathrm{YBa}_{2} \mathrm{Cu}_{3} \mathrm{O}_{6+x}$ from 1.8 to 300 K. Phys. Rev. Lett., 1993, 71, P. 1740-1743.

[13] Loram J. W. et al. Evidence on the pseudogap and condensate from the electronic specific heat. J. Phys. Chem. Solids, 2001, 62, P. 59-64.

[14] Fujita K. et al. Simultaneous Transitions in Cuprate Momentum-Space Topology and Electronic Symmetry Breaking. Science, 2014, 344, P. 612-616.

[15] Vojta M. Lattice symmetry breaking in cuprate superconductors: stripes, nematics, and superconductivity. Adv. Phys., 2009, 58, P. 699-820.

[16] Vojta M. Stripes and electronic quasiparticles in the pseudogap state of cuprate superconductors. Physica C, 2012, 481, P. 178.

[17] Sebastian S.E., Harrison N., Lonzarich G.G. Towards resolution of the Fermi surface in underdoped high- $T_{c}$ superconductors. Rep. Prog. Phys., 2012, 75, P. 102501.

[18] Abdullaev B., Park C.-H., Musakhanov M.M. Anyon bosonization of 2D fermions and single boson phase diagram implied from experiment on visualizing pair formation in superconductor $\mathrm{Bi}_{2} \mathrm{Sr}_{2} \mathrm{CaCu}_{2} \mathrm{O}_{8+\delta}$. Physica C, 2011, 471, P. 486-491.

[19] Gomes K.K. et al. Visualizing pair formation on the atomic scale in the high- $T_{c}$ superconductor $\mathrm{Bi}_{2} \mathrm{Sr}_{2} \mathrm{CaCu}_{2} \mathrm{O}_{8+\delta}$. Nature, 2007, 447, P. 569-572.

[20] Pan S.H. et al. Microscopic electronic inhomogeneity in the high- $T_{c}$ superconductor $\mathrm{Bi}_{2} \mathrm{Sr}_{2} \mathrm{CaCu}_{2} \mathrm{O}_{8+x}$. Nature, 2001, 413, P. $282-285$.

[21] Abdullaev B., Abdullaev D.B. Park C.-H., Musakhanov M.M. Intra pseudogap- and superconductivy-pair spin and charge fluctuations and underdome metal-insulator (fermion-boson)-crossover phenomena as keystones of cuprate physics. Nanosystems: Phys. Chem. Math., 2015, 6, P. 803-824.

[22] Abdullaev B., Roessler U., Musakhanov M. An analytic approach to the ground state energy of charged anyon gases. Phys. Rev. B, 2007, 76, P. 075403(1-7).

[23] March N.M., Young W.H., Sampanthar S. The Many Body Problems in Quantum Mechanics. Cambridge, University Press, 1967.

[24] Nakamae S. et al. Electronic ground state of heavily overdoped nonsuperconducting $\mathrm{La}_{2-x} \mathrm{Sr}_{x} \mathrm{CuO}_{4}$. Phys. Rev. B, 2003, 68, P. 100502(R).

[25] Smith M.F., et al. Origin of anomalous low-temperature downturns in the thermal conductivity of cuprates. Phys. Rev. B, 2005, 71, P. 014506.

[26] Luo J.L., et al. The magnetic field dependence of the electronic specific heat of $\mathrm{Y}_{0.8} \mathrm{Ca}_{0.2} \mathrm{Ba}_{2} \mathrm{Cu}_{3} \mathrm{O}_{6+x}$. ArXiv: cond-mat/0112065, 2001,7 p.

[27] Vedeneev S.I., Maude D.K. Metal-to-insulator crossover and pseudogap in single-layer $\mathrm{Bi}_{2+x} \mathrm{Sr}_{2-x} \mathrm{Cu}_{1+y} \mathrm{O}_{6+\delta}$ single crystals in high magnetic fields. Phys. Rev. B, 2004, 70, P. 184524.

[28] Landau L.D., Lifshitz E.M. Statistical Physics, Part 1. Oxford, Pergamon Press, 1980.

[29] Lifshitz E.M., Pitaevskii L.P. Statistical Physics, Part 2. Oxford, Pergamon Press, 1980.

[30] Abdullaev B. Implicit Anyon or Single Particle Boson Mechanism of HTCS and Pseudogap Regime. In Trends in Boson Research, edit. by A.V. Ling. N.Y.: Nova Science Publisher Inc., 2006, P. 139-161.

[31] Abdullaev B., Park C.-H. Bosonization of 2D Fermions due to Spin and Statistical Magnetic Field Coupling and Possible Nature of Superconductivity and Pseudogap Phases Below $E_{g}$. J. Korean Phys. Soc., 2006, 49, P. S642-S646.

[32] Ichimaru S. Plasma Physics: An Introduction to Statistical Physics of Charged Particles. New York, Benjamin/Cummings, 1986 , Chap. 7.

[33] Tallon J.L., Loram J.W. The doping dependence of $T^{*}$ - what is the real high- $T_{c}$ phase diagram? Physica C, 2001, 349, P. 53.

[34] Abrikosov A.A. Fundamentals of the Theory of Metals. Amsterdam, Elsevier Science, 1988.

[35] Daou R., et al. Linear temperature dependence of resistivity and change in the Fermi surface at the pseudogap critical point of a high- $T_{c}$ superconductor. Nature Phys., 2009, 5, P. 31.

[36] Kim D.H., Lee P.A., Wen X.-G. Massless Dirac Fermions, Gauge Fields, and Underdoped Cuprates. Phys. Rev. Lett., 1997, 79, P. 2109.

[37] Varma C.M. Theory of the pseudogap state of the cuprates. Phys. Rev. B, 2006, 73, P. 155113. 